\title{
Existence and Lyapunov Stability of Positive Periodic Solutions for a Third-Order Neutral Differential Equation
}

\author{
Jingli Ren, Zhibo Cheng, and Yueli Chen \\ Department of Mathematics, Zhengzhou University, Zhengzhou 450001, China \\ Correspondence should be addressed to Jingli Ren, renjl@zzu.edu.cn
}

Received 1 April 2011; Accepted 18 May 2011

Academic Editor: G. Scheuermann

Copyright (C) 2011 Jingli Ren et al. This is an open access article distributed under the Creative Commons Attribution License, which permits unrestricted use, distribution, and reproduction in any medium, provided the original work is properly cited.

By applying Green's function of third-order differential equation and a fixed point theorem in cones, we obtain some sufficient conditions for existence, nonexistence, multiplicity, and Lyapunov stability of positive periodic solutions for a third-order neutral differential equation.

\section{Introduction}

Neutral functional differential equations manifest themselves in many fields including biology, mechanics, and economics [1-4]. For example, in population dynamics, since a growing population consumes more (or less) food than a matured one, depending on individual species, this leads to neutral functional equations [1]. These equations also arise in classical "cobweb" models in economics where current demand depends on price but supply depends on the previous periodic solutions [2]. The study on neutral functional differential equations is more intricate than ordinary delay differential equations. In recent years, there has been a good amount of work on periodic solutions for neutral differential equations (see [5-12] and the references cited therein). For example, in [5], Wu and Wang discussed the second-order neutral delay differential equation

$$
(x(t)-c x(t-\delta))^{\prime \prime}+a(t) x(t)=\lambda b(t) f(x(t-\tau(t))) .
$$

By a fixed point theorem, they obtain some existence results of positive periodic solutions for (1.1). Recently, in [6], Cheung et al. considered second-order neutral functional differential equation

$$
(x(t)-c x(t-\tau(t)))^{\prime \prime}=a(t) x(t)-f(t, x(t-\tau(t))) .
$$


By choosing available operators and applying Krasnoselskii's fixed point theorem, they obtained sufficient conditions for the existence of periodic solutions to (1.2).

In general, most of the existing results are concentrated on first-order and secondorder neutral functional differential equations, while studies on third-order neutral functional differential equations are rather infrequent, especially on the positive periodic solutions for third-order neutral functional differential equations. In the study of high-order (in particular third-order) differential equations, the naive idea to translate the equation into a first-order differential system by defining $x_{1}=x, x_{2}=x^{\prime}, x_{3}=x^{\prime \prime}, \ldots$, works well for showing existence of periodic solutions, however, it does not obviously lead to existence proofs for positive periodic solutions, since the condition $x=x_{1} \geq 0$ of positivity for the higher order equation is different from the natural positivity condition $\left(x_{1}, x_{2}, \ldots\right) \geq 0$ for the corresponding system. Another approach, which will be used in this paper, is to transform the third-order equation into a corresponding integral equation and to establish the existence of positive periodic solutions based on a fixed point theorem in cones. Following this path one needs an explicit representation of Green's function which is rather intricate to compute. equation:

In this paper, we consider the following third-order neutral functional differential

$$
(x(t)-c x(t-\delta(t)))^{\prime \prime \prime}=-a(t) x(t)+\lambda b(t) f(x(t-\tau(t))) .
$$

Here $\lambda$ is a positive parameter; $f \in C(\mathbb{R},[0, \infty))$, and $f(x)>0$ for $x>0 ; a \in C(\mathbb{R},(0, \infty))$, $b \in C(\mathbb{R},(0, \infty)), \tau, \delta \in C^{1}(\mathbb{R}, \mathbb{R}), a(t), b(t), \delta(t)$, and $\tau(t)$ are $\omega$-periodic functions.

Notice that here neutral operator $(A x)(t)=x(t)-c x(t-\delta(t))$ is a natural generalization of the familiar operator $\left(A_{1} x\right)(t)=x(t)-c x(t-\delta)$. But $A$ possesses a more complicated nonlinearity than $A_{1}$. For example, the neutral operators $A_{1}$ is homogeneous in the following senses $\left(A_{1} x\right)^{\prime}(t)=\left(A_{1} x^{\prime}\right)(t)$, whereas the neutral operator $A$ in general is inhomogeneous. As a consequence many of the new results for differential equations with the neutral operator $A$ will not be a direct extension of known theorems for neutral differential equations.

The paper is organized as follows. In Section 2, we first analyze qualitative properties of the generalized neutral operator $A$ which will be helpful for further studies of differential equations with this neutral operator; in Section 3, we consider two types of third-order constant coefficient linear differential equations and present their Green's functions and properties for those equation; in Section 4, by an application of the fixed point index theorem we obtain sufficient conditions for the existence, multiplicity and nonexistence of positive periodic solutions to third-order neutral differential equation. We will give an example to illustrate our results; in Section 5, the Lyapunov stability of periodic solutions for the equation will then be established. And an example is also given in this section.

\section{Analysis of the Generalized Neutral Operator}

Let $X=\{x \in C(\mathbb{R}, \mathbb{R}): x(t+\omega)=x(t), t \in \mathbb{R}\}$ with norm $\|x\|=\max _{t \in[0, \omega]}|x(t)|$, and let $C_{\omega}^{+}=\{x \in C(\mathbb{R},(0, \infty)): x(t+\omega)=x(t)\}, C_{\omega}^{-}=\{x \in C(\mathbb{R},(-\infty, 0)): x(t+\omega)=x(t)\}$. Then $(X,\|\cdot\|)$ is a Banach space. A cone $K$ in $X$ is defined by $K=\{x \in X: x(t) \geq \alpha\|x\|, \forall t \in \mathbb{R}\}$, where $\alpha$ is a fixed positive number with $\alpha<1$. Moreover, define operators $A, B: X \rightarrow X$ by

$$
(A x)(t)=x(t)-c x(t-\delta(t)), \quad(B x)(t)=c x(t-\delta(t)) .
$$


Lemma 2.1. If $|c| \neq 1$, then the operator $A$ has a continuous inverse $A^{-1}$ on $X$, satisfying

(1)

$$
\left(A^{-1} f\right)(t)= \begin{cases}f(t)+\sum_{j=1}^{\infty} c^{j} f\left(s-\sum_{i=1}^{j-1} \delta\left(D_{i}\right)\right), & \text { for }|c|<1, \quad \forall f \in X, \\ -\frac{f(t+\delta(t))}{c}-\sum_{j=1}^{\infty} \frac{1}{c^{j+1}} f\left(s+\delta(t)+\sum_{i=1}^{j-1} \delta\left(D_{i}\right)\right), & \text { for }|c|>1, \quad \forall f \in X,\end{cases}
$$

(2)

$$
\left|\left(A^{-1} f\right)(t)\right| \leq \frac{\|f\|}{|1-| c||}, \quad \forall f \in X
$$

(3)

$$
\int_{0}^{\omega}\left|\left(A^{-1} f\right)(t)\right| d t \leq \frac{1}{|1-| c||} \int_{0}^{\omega}|f(t)| d t, \quad \forall f \in X .
$$

Proof. We have the following cases.

Case $1(|c|<1)$. Let $t-\delta(t)=s$ and $D_{j}=s-\sum_{i=1}^{j-1} \delta\left(D_{i}\right), j=1,2, \ldots$. Therefore

$$
\begin{gathered}
B^{j} x(t)=c^{j} x\left(s-\sum_{i=1}^{j-1} \delta\left(D_{i}\right)\right) \\
\sum_{j=0}^{\infty}\left(B^{j} f\right)(t)=f(t)+\sum_{j=1}^{\infty} c^{j} f\left(s-\sum_{i=1}^{j-1} \delta\left(D_{i}\right)\right) .
\end{gathered}
$$

Since $A=I-B$, we get from $\|B\| \leq|c|<1$ that $A$ has a continuous inverse $A^{-1}: X \rightarrow X$ with

$$
A^{-1}=(I-B)^{-1}=I+\sum_{j=1}^{\infty} B^{j}=\sum_{j=0}^{\infty} B^{j}
$$

Here $B^{0}=I$. Then

$$
\left(A^{-1} f(t)\right)=\sum_{j=0}^{\infty}\left[B^{j} f\right](t)=\sum_{j=0}^{\infty} c^{j} f\left(s-\sum_{i=1}^{j-1} \delta\left(D_{i}\right)\right)
$$


and consequently

$$
\left|\left(A^{-1} f\right)(t)\right|=\left|\sum_{j=0}^{\infty}\left[B^{j} f\right](t)\right|=\left|\sum_{j=0}^{\infty} c^{j} f\left(s-\sum_{i=1}^{j-1} \delta\left(D_{i}\right)\right)\right| \leq \frac{\|f\|}{1-|c|} .
$$

Moreover,

$$
\begin{aligned}
\int_{0}^{\omega}\left|\left(A^{-1} f\right)(t)\right| d t & =\int_{0}^{\omega}\left|\sum_{j=0}^{\infty}\left(B^{j} f\right)(t)\right| d t \\
& \leq \sum_{j=0}^{\infty} \int_{0}^{\omega}\left|\left(B^{j} f\right)(t)\right| d t \\
& =\sum_{j=0}^{\infty} \int_{0}^{\omega}\left|c^{j} f\left(s-\sum_{i=1}^{j-1} \delta\left(D_{i}\right)\right)\right| d t \\
& \leq \frac{1}{1-|c|} \int_{0}^{\omega}|f(t)| d t .
\end{aligned}
$$

Case $2(|c|>1)$. Let

$$
\begin{gathered}
E: X \longrightarrow X, \quad(E x)(t)=x(t)-\frac{1}{c} x(t+\delta(t)), \\
B_{1}: X \longrightarrow X, \quad\left(B_{1} x\right)(t)=\frac{1}{c} x(t+\delta(t)) .
\end{gathered}
$$

By definition of the linear operator $B_{1}$, we have

$$
\left(B_{1}^{j} f\right)(t)=\frac{1}{c^{j}} f\left(s+\sum_{i=1}^{j-1} \delta\left(D_{i}\right)\right)
$$

Here $D_{i}$ is defined as in Case 1. Summing over $j$ yields

$$
\sum_{j=0}^{\infty}\left(B_{1}^{j} f\right)(t)=f(t)+\sum_{j=1}^{\infty} \frac{1}{c^{j}} f\left(s+\sum_{i=1}^{j-1} \delta\left(D_{i}\right)\right)
$$

Since $\left\|B_{1}\right\|<1$, we obtain that the operator $E$ has a bounded inverse $E^{-1}$,

$$
E^{-1}: X \longrightarrow X, \quad E^{-1}=\left(I-B_{1}\right)^{-1}=I+\sum_{j=1}^{\infty} B_{1}^{j}
$$


and for all $f \in X$ we get

$$
\left(E^{-1} f\right)(t)=f(t)+\sum_{j=1}^{\infty}\left(B_{1}^{j} f\right)(t)
$$

On the other hand, from $(A x)(t)=x(t)-c x(t-\delta(t))$, we have

$$
(A x)(t)=x(t)-c x(t-\delta(t))=-c\left[x(t-\delta(t))-\frac{1}{c} x(t)\right],
$$

that is,

$$
(A x)(t)=-c(E x)(t-\delta(t)) .
$$

Let $f \in X$ be arbitrary. We are looking for $x$ such that

$$
(A x)(t)=f(t),
$$

that is

$$
-c(E x)(t-\delta(t))=f(t)
$$

Therefore

$$
(E x)(t)=-\frac{f(t+\delta(t))}{c}=: f_{1}(t)
$$

and hence

$$
x(t)=\left(E^{-1} f_{1}\right)(t)=f_{1}(t)+\sum_{j=1}^{\infty}\left(B_{1}^{j} f_{1}\right)(t)=-\frac{f(t+\delta(t))}{c}-\sum_{j=1}^{\infty} B_{1}^{j} \frac{f(t+\delta(t))}{c},
$$

proving that $A^{-1}$ exists and satisfies

$$
\begin{aligned}
{\left[A^{-1} f\right](t) } & =-\frac{f(t+\delta(t))}{c}-\sum_{j=1}^{\infty} B_{1}^{j} \frac{f(t+\delta(t))}{c} \\
& =-\frac{f(t+\delta(t))}{c}-\sum_{j=1}^{\infty} \frac{1}{c^{j+1}} f\left(s+\delta(t)+\sum_{i=1}^{j-1} \delta\left(D_{i}\right)\right) \\
\left|\left[A^{-1} f\right](t)\right| & =\left|-\frac{f(t+\delta(t))}{c}-\sum_{j=1}^{\infty} \frac{1}{c^{j+1}} f\left(s+\delta(t)+\sum_{i=1}^{j-1} \delta\left(D_{i}\right)\right)\right| \leq \frac{\|f\|}{|c|-1} .
\end{aligned}
$$

Statements (1) and (2) are proved. From the above proof, (3) can easily be deduced. 
Lemma 2.2. If $c<0$ and $|c|<\alpha$, we have for $y \in K$ that

$$
\frac{\alpha-|c|}{1-c^{2}}\|y\| \leq\left(A^{-1} y\right)(t) \leq \frac{1}{1-|c|}\|y\| .
$$

Proof. Since $c<0$ and $|c|<\alpha<1$, by Lemma 2.1, one has for $y \in K$ that

$$
\begin{aligned}
\left(A^{-1} y\right)(t) & =y(t)+\sum_{j=1}^{\infty} c^{j} y\left(s-\sum_{i=1}^{j-1} \delta\left(D_{i}\right)\right) \\
& =y(t)+\sum_{j \geq 1 \text { even }} c^{j} y\left(s-\sum_{i=1}^{j-1} \delta\left(D_{i}\right)\right)-\sum_{j \geq 1 \text { odd }}|c|^{j} y\left(s-\sum_{i=1}^{j-1} \delta\left(D_{i}\right)\right) \\
& \geq \alpha\|y\|+\alpha \sum_{j \geq 1 \text { even }} c^{j}\|y\|-\|y\| \sum_{j \geq 1 \text { odd }}|c|^{j} \\
& =\frac{\alpha}{1-c^{2}}\|y\|-\frac{|c|}{1-c^{2}}\|y\| \\
& =\frac{\alpha-|c|}{1-c^{2}}\|y\| .
\end{aligned}
$$

Lemma 2.3. If $c>0$ and $c<1$, then for $y \in K$, one has

$$
\frac{\alpha}{1-c}\|y\| \leq\left(A^{-1} y\right)(t) \leq \frac{1}{1-c}\|y\| .
$$

Proof. Since $c>0, c<1$, and $\alpha<1$, by Lemma 2.1, we have for $y \in K$ that

$$
\begin{aligned}
\left(A^{-1} y\right)(t) & =y(t)+\sum_{j \geq 1} c^{j} y\left(s-\sum_{i=1}^{j-1} \delta\left(D_{i}\right)\right) \\
& \geq \alpha\|y\|+\alpha\|y\| \sum_{j \geq 1} c^{j} \\
& =\frac{\alpha}{1-c}\|y\| .
\end{aligned}
$$

\section{Green's Functions}

Theorem 3.1. For $\rho>0$ and $h \in X$, the equation

$$
\begin{aligned}
& u^{\prime \prime \prime}-\rho^{3} u=h(t), \\
& u(0)=u(\omega), \quad u^{\prime}(0)=u^{\prime}(\omega), \quad u^{\prime \prime}(0)=u^{\prime \prime}(\omega)
\end{aligned}
$$


has a unique solution which is of the form

$$
u(t)=\int_{0}^{\omega} G_{1}(t, s)(-h(s)) \mathrm{d} s,
$$

where

$$
G_{1}(t, s)=\left\{\begin{array}{l}
\frac{2 \exp ((1 / 2) \rho(s-t))[\sin ((\sqrt{3} / 2) \rho(t-s)+\pi / 6)-w]}{3 \rho^{2}(1+\exp (-\rho \omega)-2 \exp (-\rho \omega / 2) \cos ((\sqrt{3} / 2) \rho \omega))} \\
+\frac{\exp (\rho(t-s))}{3 \rho^{2}(\exp (\rho \omega)-1)}, \quad 0 \leq s \leq t \leq \omega, \\
\frac{2 \exp ((1 / 2) \rho(s-t-\omega))[\sin ((\sqrt{3} / 2) \rho(t-s+\omega)+\pi / 6)-y]}{3 \rho^{2}(1+\exp (-\rho \omega)-2 \exp (-\rho \omega / 2) \cos ((\sqrt{3} / 2) \rho \omega))} \\
+\frac{\exp (\rho(t+\omega-s))}{3 \rho^{2}(\exp (\rho \omega)-1)}, \quad 0 \leq t \leq s \leq \omega,
\end{array}\right.
$$

where $w$ denotes $\exp (-(1 / 2) \rho \omega) \sin ((\sqrt{3} / 2) \rho(t-s-\omega)+\pi / 6)$ and $y$ denotes $\exp (-(1 /$ 2) $\rho \omega) \sin ((\sqrt{3} / 2) \rho(t-s)+\pi / 6)$.

Proof. It is easy to check that the associated homogeneous equation of (3.1) has the solution $v(t)=c_{1} \exp (\rho t)+\exp (-\rho t / 2)\left(c_{2} \cos (\sqrt{3} \rho / 2) t+c_{3} \sin (\sqrt{3} \rho / 2) t\right)$. The only periodic solution of the associated homogeneous equation of (3.1) is the trivial solution, that is, $c_{1}, c_{2}, c_{3}=0$. This follows by assuming that $v(t)$ is periodic; we immediately get that $c_{1}=0$ and by assuming that $c_{2}^{2}+c_{3}^{2}>0$ and choosing $\varphi$ such that $\sin \varphi=c_{2} / \sqrt{c_{2}^{2}+c_{3}^{2}}, \cos \varphi=c_{3} / \sqrt{c_{2}^{2}+c_{3}^{2}}$, we get

$$
\frac{v(t)}{\sqrt{c_{2}^{2}+c_{3}^{2}}}=\exp \left(-\frac{\rho t}{2}\right)\left(\sin \varphi \cos \frac{\sqrt{3} \rho}{2} t+\cos \varphi \sin \frac{\sqrt{3} \rho}{2} t\right)=\exp \left(-\frac{\rho t}{2}\right) \sin \left(\varphi+\frac{\sqrt{3} \rho}{2} t\right)
$$

which for $t \rightarrow \infty$ contradicts periodicity of $v$, proving that $c_{2}=c_{3}=0$.

Applying the method of variation of parameters, we get

$$
\begin{gathered}
c_{1}^{\prime}(t)=\frac{\exp (-\rho t)}{3 \rho^{2}} h(t), \\
c_{2}^{\prime}(t)=\frac{(\sqrt{3} / 3) \sin (\sqrt{3} \rho t / 2)-(1 / 3) \cos (\sqrt{3} \rho t / 2)}{\rho^{2}} \exp \left(\frac{\rho t}{2}\right) h(t), \\
c_{3}^{\prime}(t)=\frac{-(1 / 3) \sin (\sqrt{3} \rho t / 2)-(\sqrt{3} / 3) \cos (\sqrt{3} \rho t / 2)}{\rho^{2}} \exp \left(\frac{\rho t}{2}\right) h(t),
\end{gathered}
$$


and then

$$
\begin{gathered}
c_{1}(t)=c_{1}(0)+\int_{0}^{t} \frac{\exp (-\rho s)}{3 \rho^{2}} h(s) \mathrm{d} s, \\
c_{2}(t)=c_{2}(0)+\int_{0}^{t} \frac{(\sqrt{3} / 3) \sin (\sqrt{3} \rho s / 2)-(1 / 3) \cos (\sqrt{3} \rho s / 2)}{\rho^{2}} \exp \left(\frac{\rho s}{2}\right) h(s) \mathrm{d} s, \\
c_{3}(t)=c_{3}(0)+\int_{0}^{t} \frac{-(1 / 3) \sin (\sqrt{3} \rho s / 2)-(\sqrt{3} / 3) \cos (\sqrt{3} \rho s / 2)}{\rho^{2}} \exp \left(\frac{\rho s}{2}\right) h(s) \mathrm{d} s, \\
u(t)=c_{1}(t) \exp (\rho t)+\exp \left(-\frac{\rho t}{2}\right)\left(c_{2}(t) \cos \frac{\sqrt{3} \rho}{2} t+c_{3}(t) \sin \frac{\sqrt{3} \rho}{2} t\right) \\
=c_{1}(0) \exp (\rho t)+c_{2} \exp \left(-\frac{\rho t}{2}\right) \cos \left(\frac{\sqrt{3}}{2} \rho t\right)+c_{3}(0) \exp \left(-\frac{\rho t}{2}\right) \sin \left(\frac{\sqrt{3}}{2} \rho t\right) \\
+\int_{0}^{t} \frac{\exp (\rho(t-s))}{3 \rho^{2}} h(s) \mathrm{d} s+\int_{0}^{t} \frac{\sin ((\sqrt{3} / 2) \rho(s-t)-\pi / 6)}{6 \rho^{2}} \exp \left(\frac{\rho}{2}(s-t)\right) h(s) \mathrm{d} s .
\end{gathered}
$$

Noting that $u(0)=u(\omega), u^{\prime}(0)=u^{\prime}(\omega), u^{\prime \prime}(0)=u^{\prime \prime}(\omega)$, we obtain

$$
\begin{gathered}
c_{1}(0)=\int_{0}^{\omega} \frac{\exp (\rho(\omega-s))}{3 \rho^{2}(1-\exp (\rho \omega))} h(s) \mathrm{d} s, \\
c_{2}(0)=\int_{0}^{\omega} \frac{2 \exp (\rho(s-\omega) / 2)[\exp (-\rho \omega / 2) \sin (\pi / 6-\sqrt{3} \rho s / 2)-\sin \Phi]}{3 \rho^{2}(\exp (-\rho \omega)-2 \exp (-\rho \omega / 2) \cos (\sqrt{3} \rho \omega / 2)+1)} h(s) \mathrm{d} s, \\
c_{3}(0)=\int_{0}^{\omega} \frac{2 \exp (\rho(s-\omega) / 2)[\exp (-\rho \omega / 2) \cos (\pi / 6-\sqrt{3} \rho s / 2)-\cos \Phi]}{3 \rho^{2}(\exp (-\rho \omega)-2 \exp (-\rho \omega / 2) \cos (\sqrt{3} \rho \omega / 2)+1)} h(s) \mathrm{d} s,
\end{gathered}
$$

where $\Phi$ denotes $(\pi / 6-\sqrt{3} \rho(s-\omega) / 2)$. Therefore

$$
\begin{gathered}
u(t)=c_{1}(t) \exp (\rho t)+\exp \left(-\frac{\rho t}{2}\right)\left(c_{2}(t) \cos \frac{\sqrt{3} \rho}{2} t+c_{3}(t) \sin \frac{\sqrt{3} \rho}{2} t\right) \\
=\int_{0}^{t}\left\{\frac{2 \exp ((1 / 2) \rho(s-t))[\sin ((\sqrt{3} / 2) \rho(t-s)+\pi / 6)-w]}{3 \rho^{2}(1+\exp (-\rho \omega)-2 \exp (-\rho \omega / 2) \cos ((\sqrt{3} / 2) \rho \omega))}\right. \\
\left.+\frac{\exp (\rho(t-s))}{3 \rho^{2}(1-\exp (\rho \omega))}\right\} h(s) \mathrm{d} s
\end{gathered}
$$


ISRN Applied Mathematics

$$
\begin{aligned}
& +\int_{t}^{\omega}\left\{\frac{2 \exp ((1 / 2) \rho(s-t-\omega))[\sin ((\sqrt{3} / 2) \rho(t-s+\omega)+\pi / 6)-y]}{3 \rho^{2}(1+\exp (-\rho \omega)-2 \exp (-\rho \omega / 2) \cos ((\sqrt{3} / 2) \rho \omega))}\right. \\
& \left.\quad+\frac{\exp (\rho(t+\omega-s))}{3 \rho^{2}(1-\exp (\rho \omega))}\right\} h(s) \mathrm{d} s \\
& =\int_{0}^{\omega} G_{1}(t, s) h(s) \mathrm{d} s,
\end{aligned}
$$

where $G_{1}(t, s)$ is defined as in (3.3).

By direct calculation, we get the solution $u$ satisfies the periodic boundary value condition of the problem (3.1).

Theorem 3.2. For $\rho>0$ and $h \in X$, the equation

$$
\begin{array}{ll} 
& u^{\prime \prime \prime}+\rho^{3} u=h(t), \\
u(0)=u(\omega), & u^{\prime}(0)=u^{\prime}(\omega), \quad u^{\prime \prime}(0)=u^{\prime \prime}(\omega)
\end{array}
$$

has a unique w-periodic solution

$$
u(t)=\int_{0}^{\omega} G_{2}(t, s) h(s) \mathrm{d} s
$$

where

$$
G_{2}(t, s)=\left\{\begin{array}{l}
\frac{2 \exp ((1 / 2) \rho(t-s))[\sin ((\sqrt{3} / 2) \rho(t-s)-\pi / 6)-\mathcal{U}]}{3 \rho^{2}(1+\exp (\rho \omega)-2 \exp ((1 / 2) \rho \omega) \cos ((\sqrt{3} / 2) \rho \omega))} \\
+\frac{\exp (\rho(s-t))}{3 \rho^{2}(1-\exp (-\rho \omega))}, \quad 0 \leq s \leq t \leq \omega, \\
\frac{2 \exp ((1 / 2) \rho(t+\omega-s))[\sin ((\sqrt{3} / 2) \rho(t+\omega-s)-\pi / 6)-x]}{3 \rho^{2}(1+\exp (\rho \omega)-2 \exp ((1 / 2) \rho \omega) \cos ((\sqrt{3} / 2) \rho \omega))} \\
+\frac{\exp (\rho(s-t-\omega))}{3 \rho^{2}(1-\exp (-\rho \omega))}, \quad 0 \leq t \leq s \leq \omega .
\end{array}\right.
$$

where $u$ denotes $\exp ((1 / 2) \rho \omega) \sin ((\sqrt{3} / 2) \rho(t-s-\omega)-\pi / 6)$ and $x$ denotes $-\exp ((1 /$ 2) $\rho \omega) \sin ((\sqrt{3} / 2) \rho(t-s)-\pi / 6)$.

Proof. It is similar to the proof of Theorem 3.1 and can therefore be omitted. 
Now we present the properties of Green's functions for (3.1) and (3.9)

$$
l=\frac{1}{3 \rho^{2}(\exp (\rho \omega)-1)}, \quad L=\frac{3+2 \exp (-\rho \omega / 2)}{3 \rho^{2}(1-\exp (-\rho \omega / 2))^{2}} .
$$

Theorem 3.3. $\int_{0}^{\omega} G_{1}(t, s) \mathrm{d} s=1 / \rho^{3}$, and if $\sqrt{3} \rho \omega<(4 / 3) \pi$ holds, then $0<l<G_{1}(t, s) \leq L$ for all $t \in[0, \omega]$ and $s \in[0, \omega]$.

Proof. One has the following:

$$
\begin{gathered}
H_{1}(t, s)=\frac{\exp (\rho(t-s))}{3 \rho^{2}[\exp (\rho \omega)-1]}, \\
H_{1}^{*}(t, s)=\frac{\exp (\rho(t+\omega-s))}{3 \rho^{2}[\exp (\rho \omega)-1]}, \\
H_{2}(t, s)=\frac{2 \exp ((1 / 2) \rho(s-t))[\sin ((\sqrt{3} / 2) \rho(t-s)+\pi / 6)-w]}{3 \rho^{2}(1+\exp (-\rho \omega)-2 \exp (-\rho \omega / 2) \cos ((\sqrt{3} / 2) \rho \omega))} \\
H_{2}^{*}(t, s)=\frac{2 \exp ((1 / 2) \rho(s-t-\omega))[\sin ((\sqrt{3} / 2) \rho(t-s+\omega)+\pi / 6)-y]}{3 \rho^{2}(1+\exp (-\rho \omega)-2 \exp (-\rho \omega / 2) \cos ((\sqrt{3} / 2) \rho \omega))} .
\end{gathered}
$$

A direct computation shows that $\int_{0}^{\omega} G_{1}(t, s) \mathrm{d} s=1 / \rho^{3}$. It is easy to see that $H_{1}(t, s)>0$ for $s \in[0, t]$ and $H_{1}^{*}(t, s)>0$ for $s \in[t, \omega]$ and $\exp (-\rho \omega)+1-2 \exp (-\rho \omega / 2) \cos (\sqrt{3} \rho \omega / 2)>$ $[1-\exp (-\rho \omega / 2)]^{2}>0$.

For convenience, we denote $\theta=(\sqrt{3} / 2) \rho(t-s)+\pi / 6$

$$
\begin{aligned}
g_{1}(t, s) & =\sin \left(\frac{\sqrt{3}}{2} \rho(t-s)+\frac{\pi}{6}\right)-\exp \left(-\frac{\rho \omega}{2}\right) \sin \left(\frac{\sqrt{3}}{2} \rho(t-s-\omega)+\frac{\pi}{6}\right) \\
& =\sin (\theta)-\exp \left(-\frac{\rho \omega}{2}\right) \sin \left(\theta-\frac{\sqrt{3}}{2} \rho \omega\right), \\
g_{1}^{*}(t, s) & =\sin \left(\frac{\sqrt{3}}{2} \rho(t-s+\omega)+\frac{\pi}{6}\right)-\exp \left(-\frac{\rho \omega}{2}\right) \sin \left(\frac{\sqrt{3}}{2} \rho(t-s)+\frac{\pi}{6}\right) \\
& =\sin \left(\theta+\frac{\sqrt{3}}{2} \rho \omega\right)-\exp \left(-\frac{\rho \omega}{2}\right) \sin \theta .
\end{aligned}
$$

If $g_{1}(t, s)>0$ and $g_{1}^{*}(t, s)>0$, then obviously $H_{2}(t, s)>0, H_{2}^{*}(t, s)>0$, and $G_{1}(t, s)>0$. 
For $0 \leq s \leq t \leq \omega$, Since $\sqrt{3} \rho \omega<(4 / 3) \pi$, we have

$$
\begin{gathered}
\frac{\pi}{6} \leq \theta \leq \frac{\sqrt{3}}{2} \rho \omega+\frac{\pi}{6}<\frac{5 \pi}{6}, \\
-\frac{\pi}{2}<\frac{\pi}{6}-\frac{\sqrt{3}}{2} \rho \omega \leq \theta-\frac{\sqrt{3}}{2} \rho \omega \leq \frac{\pi}{6} .
\end{gathered}
$$

(i) For $-\pi / 2<\theta-(\sqrt{3} / 2) \rho \omega \leq 0$, then $\sin \theta>0$, $\sin (\theta-(\sqrt{3} / 2) \rho \omega)<0$, we get $g_{1}(t, s)>0$,

(ii) For $0<\theta-(\sqrt{3} / 2) \rho \omega \leq \pi / 6$, we have $\sin \theta>0, \sin (\theta-(\sqrt{3} / 2) \rho \omega)>0$, and

$$
\begin{aligned}
0<\frac{\sqrt{3}}{4} \rho \omega & \leq \theta-\frac{\sqrt{3}}{4} \rho \omega \leq \frac{\pi}{6}+\frac{\sqrt{3}}{4} \rho \omega<\frac{\pi}{2} \\
g_{1}(t, s) & =\sin (\theta)-\exp \left(-\frac{\rho \omega}{2}\right) \sin \left(\theta-\frac{\sqrt{3}}{2} \rho \omega\right) \\
& \geq \sin \theta-\sin \left(\theta-\frac{\sqrt{3}}{2} \rho \omega\right) \\
& =2 \cos \left(\theta-\frac{\sqrt{3}}{4} \rho \omega\right) \sin \left(\frac{\sqrt{3}}{4} \rho \omega\right) \\
& >0 .
\end{aligned}
$$

For $0 \leq t \leq s \leq \omega$,

$$
\begin{gathered}
-\frac{\pi}{2}<-\frac{\sqrt{3}}{2} \rho \omega+\frac{\pi}{6} \leq \theta \leq \frac{\pi}{6} \\
\frac{\pi}{6} \leq \theta+\frac{\sqrt{3}}{2} \rho \omega \leq \frac{\pi}{6}+\frac{\sqrt{3}}{2} \rho \omega<\frac{5}{6} \pi .
\end{gathered}
$$

(i) For $-\pi / 2<\theta \leq 0$, we have $\sin \theta<0, \sin (\theta+(\sqrt{3} / 2) \rho \omega)>0$, and then $g_{1}^{*}(t, s)>0$.

(ii) For $0<\theta \leq \pi / 6$, we have $\sin \theta>0, \sin (\theta+(\sqrt{3} / 2) \rho \omega)>0$, and

$$
\begin{aligned}
& 0<\theta+\frac{\sqrt{3}}{4} \rho \omega<\frac{\pi}{2} \\
g_{1}^{*}(t, s)= & \sin \left(\theta+\frac{\sqrt{3}}{2} \rho \omega\right)-\exp \left(-\frac{\rho \omega}{2}\right) \sin \theta \\
\geq & \sin \left(\theta+\frac{\sqrt{3}}{2} \rho \omega\right)-\sin \theta \\
= & 2 \cos \left(\theta+\frac{\sqrt{3}}{4} \rho \omega\right) \sin \left(\frac{\sqrt{3}}{4} \rho \omega\right) \\
> & 0 .
\end{aligned}
$$


If $\sqrt{3} \rho \omega<(4 / 3) \rho \omega$, we get $g_{1}(t, s)>0$ and $g_{1}^{*}(t, s)>0$, proving that $G(t, s)>0$ for all $t \in[0, \omega]$ and $s \in[0, \omega]$.

Next we compute a lower and an upper bound for $G_{1}(t, s)$ for $s \in[0, \omega]$. We have

$$
\begin{aligned}
l & =\frac{1}{3 \rho^{2}(\exp (\rho \omega)-1)} \\
& \leq \frac{\exp (\rho(t+\omega-s))}{3 \rho^{2}(\exp (\rho \omega)-1)}<G_{1}(t, s) \\
& \leq \frac{\exp (\rho(t+\omega-s))}{3 \rho^{2}[\exp (\rho \omega)-1]}+\frac{\exp (\rho(s-t-\omega) / 2)[2+2 \exp (-\rho \omega / 2)]}{3 \rho^{2}[\exp (-\rho \omega)+1-2 \exp (-\rho \omega / 2) \cos (\sqrt{3} \rho \omega / 2)]} \\
& \leq \frac{\exp (\rho \omega)}{3 \rho^{2}[\exp (\rho \omega)-1]}+\frac{2+2 \exp (-\rho \omega / 2)}{3 \rho^{2}[\exp (-\rho \omega)+1-2 \exp (-\rho \omega / 2) \cos (\sqrt{3} \rho \omega / 2)]} \\
& \leq \frac{1}{3 \rho^{2}[1-\exp (-\rho \omega)]}+\frac{2+2 \exp (-\rho \omega / 2)}{3 \rho^{2}[1-\exp (-\rho \omega / 2)]^{2}} \\
& \leq \frac{3+2 \exp (-\rho \omega / 2)}{3 \rho^{2}[1-\exp (-\rho \omega / 2)]^{2}}=L .
\end{aligned}
$$

The proof is complete.

Similarly, the following dual theorem can be proved.

Theorem 3.4. $\int_{0}^{\omega} G_{2}(t, s) \mathrm{d} s=1 / \rho^{3}$, and if $\sqrt{3} \rho \omega<(4 / 3) \pi$ holds, then $0<l<G_{2}(t, s) \leq L$ for all $[0, \omega]$ and $s \in[0, \omega]$.

\section{Positive Periodic Solutions for (1.3)}

Define the Banach space $X$ as in Section 2. Denote

$$
\begin{gathered}
M=\max \{a(t): t \in[0, \omega]\}, \quad m=\min \{a(t): t \in[0, \omega]\}, \quad \rho^{3}=M, \\
k=l(M+m)+L M, \quad k_{1}=\frac{k-\sqrt{k^{2}-4 L l M m}}{2 L M}, \quad \alpha=\frac{l[m-(M+m)|c|]}{L M(1-|c|)} .
\end{gathered}
$$

It is easy to see that $M, m, \beta, L, l, k, k_{1}>0$.

Now we consider (1.3). First let

$$
\bar{f}_{0}=\varlimsup_{x \rightarrow 0} \frac{f(x)}{x}, \quad \bar{f}_{\infty}=\varlimsup_{x \rightarrow \infty} \frac{f(x)}{x}, \quad \underline{f}_{0}=\underline{\lim _{x \rightarrow 0}} \frac{f(x)}{x}, \quad \underline{f}_{\infty}=\underline{\lim _{x \rightarrow \infty}} \frac{f(x)}{x},
$$


and denote

$$
\begin{array}{ll}
\bar{i}_{0}: \text { number of } 0^{\prime} \text { s in }\left(\bar{f}_{0}, \bar{f}_{\infty}\right), & \underline{i}_{-} \text {: number of } 0^{\prime} \text { s in }\left(\underline{f}_{0^{\prime}} \underline{f}_{-\infty}\right), \\
\bar{i}_{\infty}: \text { number of } \infty^{\prime} \text { s in }\left(\bar{f}_{0}, \bar{f}_{\infty}\right), & \underline{i}_{\infty} \text { : numberof } \infty^{\prime} \text { s in }\left(\underline{f}_{0^{\prime}} \underline{f}_{\infty}\right) .
\end{array}
$$

It is clear that $\bar{i}_{0}, \underline{i}_{0}, \bar{i}_{\infty}, \underline{i}_{-\infty} \in\{0,1,2\}$. We will show that (1.3) has $\bar{i}_{0}$ or $\underline{i}_{-\infty}$ positive $w$-periodic solutions for sufficiently large or small $\lambda$, respectively.

In the following we discuss (1.3) in two cases, namely, the case where $c<0$, and $c>-\min \left\{k_{1}, m /(M+m)\right\}$ (note that $c>-m /(M+m)$ implies $\alpha>0, c>-k_{1}$ implies $|c|<\alpha$ ); and the case where $c>0$ and $c<\min \{m /(M+m),(L M-l m) /((L-l) M-l m)\}$, (note that $c<m /(M+m)$ implies $\alpha>0, c<(L M-l m) /((L-l) M-l m)$ implies $\alpha<1)$. Obviously, we have $|c|<1$ which makes Lemma 2.1 applicable for both cases, and also Lemmas 2.2 or 2.3, respectively.

Let $K=\{x \in X: x(t) \geq \alpha\|x\|\}$ denote the cone in $X$, where $\alpha$ is just as defined above. We also use $K_{r}=\{x \in K:\|x\|<r\}$ and $\partial K_{r}=\{x \in K:\|x\|=r\}$.

Let $y(t)=(A x)(t)$, then from Lemma 2.1 we have $x(t)=\left(A^{-1} y\right)(t)$. Hence (1.3) can be transformed into

$$
y^{\prime \prime \prime}(t)+a(t)\left(A^{-1} y\right)(t)=\lambda b(t) f\left(\left(A^{-1} y\right)(t-\tau(t))\right)
$$

which can be further rewritten as

$$
y^{\prime \prime \prime}(t)+a(t) y(t)-a(t) H(y(t))=\lambda b(t) f\left(\left(A^{-1} y\right)(t-\tau(t))\right)
$$

where $H(y(t))=y(t)-\left(A^{-1} y\right)(t)=-c\left(A^{-1} y\right)(t-\delta(t))$.

Now we discuss the two cases separately.

4.1. Case I: $c<0$ and $c>-\min \left\{k_{1}, m /(M+m)\right\}$

Now we consider

$$
y^{\prime \prime \prime}(t)+a(t) y(t)-a(t) H(y(t))=h(t), \quad h \in C_{\omega}^{+},
$$

and define operators $T, \widehat{H}: X \rightarrow X$ by

$$
(T h)(t)=\int_{t}^{t+\omega} G_{2}(t, s) h(s) \mathrm{ds}, \quad(\widehat{\mathrm{H} y})(\mathrm{t})=\mathrm{M}-\mathrm{a}(\mathrm{t}) \mathrm{y}(\mathrm{t})+\mathrm{a}(\mathrm{t}) \mathrm{H}(\mathrm{y}(\mathrm{t})) .
$$

Clearly $T, \widehat{H}$ are completely continuous, $(T h)(t)>0$ for $h(t)>0$ and $\|\widehat{H}\| \leq(M-m+$ $M(|c| /(1-|c|)))$. By Theorem 3.2, the solution of (4.6) can be written in the following form:

$$
y(t)=(T h)(t)+(T \widehat{H} y)(t)
$$


In view of $c<0$ and $c>-\min \left\{k_{1}, m /(M+m)\right\}$, we have

$$
\|T \widehat{H}\| \leq\|T\|\|\widehat{H}\| \leq \frac{M-m+m|c|}{M(1-|c|)}<1,
$$

and hence

$$
y(t)=(I-T \widehat{H})^{-1}(T h)(t) .
$$

Define an operator $P: X \rightarrow X$ by

$$
(P h)(t)=(I-T \widehat{H})^{-1}(T h)(t) .
$$

Obviously, for any $h \in C_{\omega}^{+}$, if $(\sqrt{3} / 2) \rho \omega<\pi$ hold, $y(t)=(P h)(t)$ is the unique positive $\omega$-periodic solution of (4.6).

Lemma 4.1. $P$ is completely continuous, and

$$
(T h)(t) \leq(P h)(t) \leq \frac{M(1-|c|)}{m-(M+m)|c|}\|T h\|, \quad \forall h \in C_{\omega}^{+} .
$$

Proof. By the Neumann expansion of $P$, we have

$$
\begin{aligned}
P & =(I-T \widehat{H})^{-1} T \\
& =\left(I+T \widehat{H}+(T \widehat{H})^{2}+\cdots+(T \widehat{H})^{n}+\cdots\right) T \\
& =T+T \widehat{H} T+(T \widehat{H})^{2} T+\cdots+(T \widehat{H})^{n} T+\cdots
\end{aligned}
$$

Since $T$ and $\widehat{H}$ are completely continuous, so is $P$. Moreover, by (4.13) and recalling that $\|T \widehat{H}\| \leq(M-m+m|c|) / M(1-|c|)<1$, we get

$$
(T h)(t) \leq(P h)(t) \leq \frac{M(1-|c|)}{m-(M+m)|c|}\|T h\| .
$$

Define an operator $Q: X \rightarrow X$ by

$$
Q y(t)=P\left(\lambda b(t) f\left(\left(A^{-1} y\right)(t-\tau(t))\right)\right)
$$

Lemma 4.2. One has that $Q(K) \subset K$. 
Proof. From the definition of $Q$, it is easy to verify that $Q y(t+\omega)=Q y(t)$. For $y \in K$, we have from Lemma 4.1 that

$$
\begin{aligned}
Q y(t) & =P\left(\lambda b(t) f\left(\left(A^{-1} y\right)(t-\tau(t))\right)\right) \\
& \geq T\left(\lambda b(t) f\left(\left(A^{-1} y\right)(t-\tau(t))\right)\right) \\
& =\lambda \int_{t}^{t+\omega} G_{2}(t, s) b(s) f\left[\left(A^{-1} y\right)(s-\tau(s))\right] \mathrm{d} s \\
& \geq \lambda l \int_{0}^{\omega} b(s) f\left[\left(A^{-1} y\right)(s-\tau(s))\right] \mathrm{d} s .
\end{aligned}
$$

On the other hand,

$$
\begin{aligned}
Q y(t) & =P\left(\lambda b(t) f\left(\left(A^{-1} y\right)(t-\tau(t))\right)\right) \\
& \leq \frac{M(1-|c|)}{m-(M+m)|c|}\left\|T\left(\lambda b(t) f\left(\left(A^{-1} y\right)(t-\tau(t))\right)\right)\right\| \\
& =\lambda \frac{M(1-|c|)}{m-(M+m)|c|} \max _{t \in[0, \omega]} \int_{t}^{t+\omega} G_{2}(t, s) b(s) f\left(\left(A^{-1} y\right)(s-\tau(s))\right) \mathrm{d} s \\
& \leq \lambda \frac{M(1-|c|)}{m-(M+m)|c|} L \int_{0}^{\omega} b(s) f\left(\left(A^{-1} y\right)(s-\tau(s))\right) \mathrm{d} s .
\end{aligned}
$$

Therefore

$$
Q y(t) \geq \frac{l[m-(M+m)|c|]}{L M(1-|c|)}\|Q y\|=\alpha\|Q y\|
$$

that is, $Q(K) \subset K$.

From the continuity of $P$, it is easy to verify that $Q$ is completely continuous in $X$. Comparing (4.5) to (4.6), it is obvious that the existence of periodic solutions for (4.5) is equivalent to the existence of fixed points for the operator $Q$ in $X$. Recalling Lemma 4.2, the existence of positive periodic solutions for (4.5) is equivalent to the existence of fixed points of $Q$ in $K$. Furthermore, if $Q$ has a fixed point $y$ in $K$, it means that $\left(A^{-1} y\right)(t)$ is a positive $\omega$-periodic solutions of (1.3).

Lemma 4.3. If there exists $\eta>0$ such that

$$
f\left(\left(A^{-1} y\right)(t-\tau(t))\right) \geq\left(A^{-1} y\right)(t-\tau(t)) \eta, \quad \text { for } t \in[0, \omega], y \in K,
$$

then

$$
\|Q y\| \geq \lambda \ln \frac{\alpha-|c|}{1-c^{2}} \int_{0}^{\omega} b(s) \mathrm{d} s\|y\|, \quad y \in K
$$


Proof. By Lemma 2.2, Theorem 3.4, and Lemma 4.1, we have for $y \in K$ that

$$
\begin{aligned}
Q y(t) & =P\left(\lambda b(t) f\left(\left(A^{-1} y\right)(t-\tau(t))\right)\right) \\
& \geq T\left(\lambda b(t) f\left(\left(A^{-1} y\right)(t-\tau(t))\right)\right) \\
& =\lambda \int_{t}^{t+\omega} G_{2}(t, s) b(s) f\left(\left(A^{-1} y\right)(s-\tau(s))\right) \mathrm{d} s \\
& \geq \lambda l \eta \int_{0}^{\omega} b(s)\left(A^{-1} y\right)(s-\tau(s)) \mathrm{d} s \\
& \geq \lambda \ln \frac{\alpha-|c|}{1-c^{2}} \int_{0}^{\omega} b(s) \mathrm{d} s\|y\| .
\end{aligned}
$$

Hence

$$
\|Q y\| \geq \lambda l \eta \frac{\alpha-|c|}{1-c^{2}} \int_{0}^{\omega} b(s) \mathrm{d} s\|y\|, \quad y \in K
$$

Lemma 4.4. If there exists $\varepsilon>0$ such that

$$
f\left(\left(A^{-1} y\right)(t-\tau(t))\right) \leq\left(A^{-1} y\right)(t-\tau(t)) \varepsilon, \quad \text { for } t \in[0, \omega], y \in K
$$

then

$$
\|Q y\| \leq \lambda \varepsilon \frac{L M \int_{0}^{\omega} b(s) d s}{m-(M+m)|c|}\|y\|, \quad y \in K
$$

Proof. By Lemma 2.2, Theorem 3.4, and Lemma 4.1, we have

$$
\begin{aligned}
\|Q y(t)\| & \leq \lambda \frac{M(1-|c|)}{m-(M+m)|c|} L \int_{0}^{\omega} b(s) f\left(\left(A^{-1} y\right)(s-\tau(s))\right) \mathrm{d} s \\
& \leq \lambda \frac{M(1-|c|)}{m-(M+m)|c|} L \varepsilon \int_{0}^{\omega} b(s)\left(A^{-1} y\right)(s-\tau(s)) \mathrm{d} s \\
& \leq \lambda \varepsilon \frac{L M \int_{0}^{\omega} b(s) \mathrm{d} s}{m-(M+m)|c|}\|y\| .
\end{aligned}
$$

Define

$$
\begin{gathered}
F(r)=\max \left\{f(t): 0 \leq t \leq \frac{r}{1-|c|}\right\}, \\
f_{1}(r)=\min \left\{f(t): \frac{\alpha-|c|}{1-c^{2}} r \leq t \leq \frac{r}{1-|c|}\right\} .
\end{gathered}
$$


Lemma 4.5. If $y \in \partial K_{r}$, then

$$
\|Q y\| \geq \lambda l f_{1}(r) \int_{0}^{\omega} b(s) \mathrm{ds}\|\mathrm{y}\|
$$

Proof. By Lemma 2.2, we obtain $\left((\alpha-|c|) /\left(1-c^{2}\right)\right) r \leq\left(A^{-1} y\right)(t-\tau(t)) \leq r /(1-|c|)$ for $y \in \partial K_{r}$, which yields $f\left(\left(A^{-1} y\right)(t-\tau(t))\right) \geq f_{1}(r)$. The Lemma now follows analog to the proof of Lemma 4.3.

Lemma 4.6. If $y \in \partial K_{r}$, then

$$
\|Q y\| \leq \lambda \frac{L M(1-|c|) F(r)}{m-(M+m)|c|} \int_{0}^{\omega} b(s) \mathrm{ds}\|\mathrm{y}\| .
$$

Proof. By Lemma 2.2, we can have $0 \leq\left(A^{-1} y\right)(t-\tau(t)) \leq r /(1-|c|)$ for $y \in \partial K_{r}$, which yields $f\left(\left(A^{-1} y\right)(t-\tau(t))\right) \leq F(r)$. Similar to the proof of Lemma 4.4, we get the conclusion.

We quote the fixed point theorem on which our results will be based.

Lemma 4.7 (see [13]). Let $X$ be a Banach space and $K$ a cone in $X$. For $r>0$, define $K_{r}=\{u \in K$ : $\|u\|<r\}$. Assume that $T: \bar{K}_{r} \rightarrow K$ is completely continuous such that $T x \neq x$ for $x \in \partial K_{r}=\{u \in$ $K:\|u\|=r\}$.

(i) If $\|T x\| \geq\|x\|$ for $x \in \partial K_{r}$, then $i\left(T, K_{r}, K\right)=0$.

(ii) If $\|T x\| \leq\|x\|$ for $x \in \partial K_{r}$, then $i\left(T, K_{r}, K\right)=1$.

Now we give our main results on positive periodic solutions for (1.3).

Theorem 4.8. (a) If $\bar{i}_{0}=1$ or 2 , then (1.3) has $\bar{i}_{0}$ positive w-periodic solutions for $\lambda>$ $1 / f_{1}(1) l \int_{0}^{\omega} b(s) \mathrm{ds}>0$,

(b) if $\underline{i}_{\infty}=1$ or 2 , then (1.3) has $\underline{i}_{\infty}$ positive $\omega$-periodic solutions for $0<\lambda<(m-(M+$ $m)|c|) / L M(1-|c|) F(1) \int_{0}^{\omega} b(s) \mathrm{ds}$,

(c) if $\bar{i}_{\infty}=0$ or $\underline{i}_{0}=0$, then (1.3) has no positive $\omega$-periodic solutions for sufficiently small or sufficiently large $\lambda>0$, respectively.

Proof. (a) Choose $r_{1}=1$. Taking $\lambda_{0}=1 / f_{1}\left(r_{1}\right) l \int_{0}^{\omega} b(s) \mathrm{ds}>0$, then for all $\lambda>\lambda_{0}$, we have from Lemma 4.5 that

$$
\|Q y\|>\|y\|, \quad \text { for } y \in \partial K_{r_{1}}
$$

Case 1. If $\bar{f}_{0}=0$, we can choose $0<\bar{r}_{2}<r_{1}$, so that $f(u) \leq \varepsilon u$ for $0 \leq u \leq \bar{r}_{2}$, where the constant $\varepsilon>0$ satisfies

$$
\lambda \varepsilon \frac{L M \int_{0}^{\omega} b(s) \mathrm{d} s}{m-(M+m)|c|}<1 .
$$


Letting $r_{2}=(1-|c|) \bar{r}_{2}$, we have $f\left(\left(A^{-1} y\right)(t-\tau(t))\right) \leq \varepsilon\left(A^{-1} y\right)(t-\tau(t))$ for $y \in K_{r_{2}}$. By Lemma 2.2, we have $0 \leq\left(A^{-1} y\right)(t-\tau(t)) \leq\|y\| /(1-|c|) \leq \bar{r}_{2}$ for $y \in \partial K_{r_{2}}$. In view of Lemma 4.4 and (4.30), we have for $y \in \partial K_{r_{2}}$ that

$$
\|Q y\| \leq \lambda \varepsilon \frac{L M \int_{0}^{\omega} b(s) \mathrm{d} s}{m-(M+m)|c|}\|y\|<\|y\|
$$

It follows from Lemma 4.7 and (4.29) that

$$
i\left(Q, K_{r_{2}}, K\right)=1, \quad i\left(Q, K_{r_{1}}, K\right)=0 .
$$

thus $i\left(Q, K_{r_{1}} \backslash \bar{K}_{r_{2}}, K\right)=-1$ and $Q$ has a fixed point $y$ in $K_{r_{1}} \backslash \bar{K}_{r_{2}}$, which means $\left(A^{-1} y\right)(t)$ is a positive $\omega$-positive solution of (1.3) for $\lambda>\lambda_{0}$.

Case 2. If $\bar{f}_{\infty}=0$, there exists a constant $\widetilde{H}>0$ such that $f(u) \leq \varepsilon u$ for $u \geq \widetilde{H}$, where the constant $\varepsilon>0$ satisfies

$$
\lambda \varepsilon \frac{L M \int_{0}^{\omega} b(s) \mathrm{ds}}{m-(M+m)|c|}<1 .
$$

Letting $r_{3}=\max \left\{2 r_{1}, \widetilde{H}\left(1-c^{2}\right) /(\alpha-|c|)\right\}$, we have $f\left(\left(A^{-1} y\right)(t-\tau(t))\right) \leq \varepsilon\left(A^{-1} y\right)(t-\tau(t))$ for $y \in K_{r_{3}}$. By Lemma 2.2, we have $\left(A^{-1} y\right)(t-\tau(t)) \geq\left((\alpha-|c|) /\left(1-c^{2}\right)\right)\|y\| \geq \widetilde{H}$ for $y \in \partial K_{r_{3}}$. Thus by Lemma 4.4 and (4.33), we have for $y \in \partial K_{r_{3}}$ that

$$
\|Q y\| \leq \lambda \varepsilon \frac{L M \int_{0}^{\omega} b(s) \mathrm{ds}}{m-(M+m)|c|}\|y\|<\|y\| .
$$

Recalling Lemma 4.7 and (4.29) and that

$$
i\left(Q, K_{r_{3}}, K\right)=1, \quad i\left(Q, K_{r_{1}}, K\right)=0,
$$

then $i\left(Q, K_{r_{3}} \backslash \bar{K}_{r_{1}}, K\right)=1$ and $Q$ has a fixed point $y$ in $K_{r_{3}} \backslash \bar{K}_{r_{1}}$, which means $\left(A^{-1} y\right)(t)$ is a positive $\omega$-positive solution of (1.3) for $\lambda>\lambda_{0}$.

Case 3. If $\bar{f}_{0}=\bar{f}_{\infty}=0$, from the above arguments, there exist $0<r_{2}<r_{1}<r_{3}$ such that $Q$ has a fixed point $y_{1}(t)$ in $K_{r_{1}} \backslash \bar{K}_{r_{2}}$ and a fixed point $y_{2}(t)$ in $K_{r_{3}} \backslash \bar{K}_{r_{1}}$. Consequently, $\left(A^{-1} y_{1}\right)(t)$ and $\left(A^{-1} y_{2}\right)(t)$ are two positive $\omega$-periodic solutions of (1.3) for $\lambda>\lambda_{0}$.

(b) Let $r_{1}=1$. Taking $\lambda_{0}=(m-(M+m)|c|) / L M(1-|c|) F\left(r_{1}\right) \int_{0}^{\omega} b(s) \mathrm{d} s>0$, then by Lemma 4.6 we know if $\lambda<\lambda_{0}$, then

$$
\|Q y\|<\|y\|, \quad y \in \partial K_{r_{1}}
$$


Case 1. If $\underline{f}_{0}=\infty$, we can choose $0<\bar{r}_{2}<r_{1}$ so that $f(u) \geq \eta u$ for $0 \leq u \leq \bar{r}_{2}$, where the constant $\eta>0$ satisfies

$$
\lambda l \eta \frac{\alpha-|c|}{1-c^{2}} \int_{0}^{\omega} b(s) \mathrm{d} s>1
$$

Letting $r_{2}=(1-|c|) \bar{r}_{2}$, we have $f\left(\left(A^{-1} y\right)(t-\tau(t))\right) \geq \eta\left(A^{-1} y\right)(t-\tau(t))$ for $y \in K_{r_{2}}$. By Lemma 2.2, we have $0 \leq\left(A^{-1} y\right)(t-\tau(t)) \leq\|y\| /(1-|c|) \leq \bar{r}_{2}$ for $y \in \partial K_{r_{2}}$. Thus by Lemma 4.3 and (4.37),

$$
\|Q y\| \geq \lambda l \eta \frac{\alpha-|c|}{1-c^{2}} \int_{0}^{\omega} b(s) \mathrm{ds}\|\mathrm{y}\|>\|\mathrm{y}\|
$$

It follows from Lemma 4.7 and (4.36) that

$$
i\left(Q, K_{r_{2}}, K\right)=0, \quad i\left(Q, K_{r_{1}}, K\right)=1,
$$

which implies $i\left(Q, K_{r_{1}} \backslash \bar{K}_{r_{2}}, K\right)=1$ and $Q$ has a fixed point $y$ in $K_{r_{1}} \backslash \bar{K}_{r_{2}}$. Therefore $\left(A^{-1} y\right)(t)$ is a positive $\omega$-periodic solution of (1.3) for $0<\lambda<\lambda_{0}$.

Case 2. If $\underline{f}_{\infty}=\infty$, there exists a constant $\widetilde{H}>0$ such that $f(u) \geq \eta u$ for $u \geq \widetilde{H}$, where the constant $\eta>0$ satisfies

$$
\lambda l \eta \frac{\alpha-|c|}{1-c^{2}} \int_{0}^{\omega} b(s) \mathrm{d} s>1
$$

Let $r_{3}=\max \left\{2 r_{1}, \widetilde{H}\left(1-c^{2}\right) /(\alpha-|c|)\right\}$, we have $f\left(\left(A^{-1} y\right)(t-\tau(t))\right) \geq \eta\left(A^{-1} y\right)(t-\tau(t))$ for $y \in K_{r_{3}}$. By Lemma 2.2, we have $\left(A^{-1} y\right)(t-\tau(t)) \geq\left((\alpha-|c|) /\left(1-c^{2}\right)\right)\|y\| \geq \widetilde{H}$ for $y \in \partial K_{r_{3}}$. Thus by Lemma 4.3 and (4.40), we have for $y \in \partial K_{r_{3}}$ that

$$
\|Q y\| \geq \lambda \ln \frac{\alpha-|c|}{1-c^{2}} \int_{0}^{\omega} b(s) \mathrm{ds}\|\mathrm{y}\|>\|\mathrm{y}\|
$$

It follows from Lemma 4.7 and (4.36) that

$$
i\left(Q, K_{r_{3}}, K\right)=0, \quad i\left(Q, K_{r_{1}}, K\right)=1,
$$

that is, $i\left(Q, K_{r_{3}} \backslash \bar{K}_{r_{1}}, K\right)=-1$ and $Q$ has a fixed point $y$ in $K_{r_{3}} \backslash \bar{K}_{r_{1}}$. That means $\left(A^{-1} y\right)(t)$ is a positive $\omega$-periodic solution of (1.3) for $0<\lambda<\lambda_{0}$.

Case 3. If $\underline{f}_{0}=\underline{f}_{\infty}=\infty$, from the above arguments, $Q$ has a fixed point $y_{1}$ in $K_{r_{1}} \backslash \bar{K}_{r_{2}}$ and a fixed point $y_{2}$ in $K_{r_{3}} \backslash \bar{K}_{r_{1}}$. Consequently, $\left(A^{-1} y_{1}\right)(t)$ and $\left(A^{-1} y_{2}\right)(t)$ are two positive $\omega$ periodic solutions of (1.3) for $0<\lambda<\lambda_{0}$. 
(c) By Lemma 2.2, if $y \in K$, then $\left(A^{-1} y\right)(t-\tau(t)) \geq\left((\alpha-|c|) /\left(1-c^{2}\right)\right)\|y\|>0$ for $t \in[0, \omega]$.

Case 1. If $\underline{i}_{0}=0$, we have $\underline{f}_{0}>0$ and $\underline{f}_{\infty}>0$. Letting $b_{1}=\min \{f(u) / u ; u>0\}>0$, then we obtain

$$
f(u) \geq b_{1} u, \quad u \in[0,+\infty)
$$

Assume $y(t)$ is a positive $\omega$-periodic solution of (1.3) for $\lambda>\lambda_{0}$, where $\lambda_{0}=\left(1-c^{2}\right) / l b_{1}(\alpha-$ $|c|) \int_{0}^{\omega} b(s) \mathrm{ds}>0$. Since $Q y(t)=y(t)$ for $t \in[0, \omega]$, then by Lemma 4.3, if $\lambda>\lambda_{0}$ we have

$$
\|y\|=\|Q y\| \geq \lambda l b_{1} \frac{\alpha-|c|}{1-c^{2}} \int_{0}^{\omega} b(s) \mathrm{ds}\|\mathrm{y}\|>\|\mathrm{y}\|
$$

which is a contradiction.

Case 2. If $\bar{i}_{\infty}=0$, we have $\bar{f}_{0}<\infty$ and $\bar{f}_{\infty}<\infty$. Letting $b_{2}=\max \{f(u) / u: u>0\}>0$, then we obtain

$$
f(u) \leq b_{2} u, \quad u \in[0, \infty)
$$

Assume $y(t)$ is a positive $\omega$-periodic solution of (1.3) for $0<\lambda<\lambda_{0}$, where $\lambda_{0}=(m-(M+$ $m)|c|) / b_{2} L M \int_{0}^{\omega} b(s)$ ds. Since $Q y(t)=y(t)$ for $t \in[0, \omega]$, it follows from Lemma 4.4 that

$$
\|y\|=\|Q y\| \leq \lambda b_{2} \frac{L M \int_{0}^{\omega} b(s) \mathrm{ds}}{m-(M+m)|c|}\|y\|<\|y\|,
$$

which is a contradiction.

Theorem 4.9. (a) If there exists a constant $b_{1}>0$ such that $f(u) \geq b_{1} u$ for $u \in[0,+\infty)$, then (1.3) has no positive $\omega$-periodic solution for $\lambda>\left(1-c^{2}\right) / l b_{1}(\alpha-|c|) \int_{0}^{\omega} b(s) \mathrm{ds}$.

(b) If there exists a constant $b_{2}>0$ such that $f(u) \leq b_{2} u$ for $u \in[0,+\infty)$, then (1.3) has no positive $\omega$-periodic solution for $0<\lambda<(m-(M+m)|c|) / b_{2} L M \int_{0}^{\omega} b(s) \mathrm{d}$.

Proof. From the proof of (c) in Theorem 4.8, we obtain this theorem immediately.

Theorem 4.10. Assume that $\underline{i}_{0}=\bar{i}_{0}=\underline{i}_{\infty}=\bar{i}_{\infty}=0$ and that one of the following conditions holds:
(1) $\bar{f}_{0} \leq \underline{f}_{\infty} ;$
(2) $\underline{f}_{0}>\bar{f}_{\infty}$;
(3) $\underline{f}_{0} \leq \underline{f}_{\infty} \leq \bar{f}_{0} \leq \bar{f}_{\infty}$;
(4) $\underline{f}_{\infty} \leq \underline{f}_{0} \leq \bar{f}_{\infty} \leq \bar{f}_{0}$. 
If

$$
\frac{1-c^{2}}{l(\alpha-|c|) \int_{0}^{\omega} b(s) \mathrm{ds} \max \left\{\underline{\mathrm{f}}_{0}, \overline{\mathrm{f}}_{0}, \underline{\mathrm{f}}_{-\infty}, \overline{\mathrm{f}}_{\infty}\right\}}<\lambda<\frac{m-(M+m)|c|}{L M \int_{0}^{\omega} b(s) \mathrm{ds} \min \left\{\underline{\mathrm{f}}_{0}, \overline{\mathrm{f}}_{0}, \underline{\mathrm{f}}_{-\infty}, \overline{\mathrm{f}}_{\infty}\right\}}
$$

then (1.3) has one positive w-periodic solution.

Proof. We have the following cases.

Case 1. If $\bar{f}_{0} \leq \underline{f}_{\infty}$, then

$$
\frac{1-c^{2}}{\bar{f}_{\infty} l(\alpha-|c|) \int_{0}^{\omega} b(s) \mathrm{ds}}<\lambda<\frac{m-(M+m)|c|}{\underline{f}_{0} L M \int_{0}^{\omega} b(s) \mathrm{ds}}
$$

It is easy to see that there exists a $0<\varepsilon<f_{\infty}$ such that

$$
\frac{1-c^{2}}{\left(\bar{f}_{\infty}-\varepsilon\right) l(\alpha-|c|) \int_{0}^{\omega} b(s) \mathrm{ds}}<\lambda<\frac{m-(M+m)|c|}{\left(\underline{f}_{0}+\varepsilon\right) L M \int_{0}^{\omega} b(s) \mathrm{ds}} .
$$

For the above $\varepsilon$, we choose $\bar{r}_{1}>0$ such that $f(u) \leq\left(\underline{f}_{0}+\varepsilon\right) u$ for $0 \leq u \leq \bar{r}_{1}$. Letting $r_{1}=$ $(1-|c|) \bar{r}_{1}$, we have $f\left(\left(A^{-1} y\right)(t-\tau(t))\right) \leq\left(\underline{f}_{0}+\varepsilon\right)\left(A^{-1} y\right)(t-\tau(t))$ for $y \in K_{r_{1}}$. By Lemma 2.2, we have $0 \leq\left(A^{-1} y\right)(t-\tau(t)) \leq\|y\| /(1-|c|) \leq \bar{r}_{1}$ for $K \in \partial K_{r_{1}}$. Thus by Lemma 4.4 we have for $y \in \partial K_{r_{1}}$ that

$$
\|Q y\| \leq \lambda\left(\underline{f}_{0}+\varepsilon\right) \frac{L M \int_{0}^{\omega} b(s) \mathrm{ds}}{m-(M+m)|c|}\|y\|<\|y\|
$$

On the other hand, there exists a constant $\widetilde{H}>0$ such that $f(u) \geq\left(\bar{f}_{\infty}-\varepsilon\right) u$ for $u \geq \widetilde{H}$. Letting $r_{2}=\max \left\{2 r_{1}, \widetilde{H}\left(1-c^{2}\right) /(\alpha-|c|)\right\}$, we have $f\left(\left(A^{-1} y\right)(t-\tau(t))\right) \geq\left(\bar{f}_{\infty}-\varepsilon\right)\left(A^{-1} y\right)(t-\tau(t))$ for $y \in K_{r_{2}}$. By Lemma 2.2, we have $\left(A^{-1} y\right)(t-\tau(t)) \geq\left((\alpha-|c|) /\left(1-c^{2}\right)\right)\|y\| \geq \widetilde{H}$ for $y \in \partial K_{r_{2}}$. Thus by Lemma 4.3, for $y \in \partial K_{r_{2}}$,

$$
\|Q y\| \geq \lambda l\left(\bar{f}_{\infty}-\varepsilon\right) \frac{\alpha-|c|}{1-c^{2}} \int_{0}^{\omega} b(s) \mathrm{ds}\|\mathrm{y}\|>\|\mathrm{y}\| .
$$

It follows from Lemma 4.7 that

$$
i\left(Q, K_{r_{1}}, K\right)=1, \quad i\left(Q, K_{r_{2}}, K\right)=0
$$

Thus $i\left(Q, K_{r_{2}} \backslash \bar{K}_{r_{1}}, K\right)=-1$ and $Q$ has a fixed point $y$ in $K_{r_{2}} \backslash \bar{K}_{r_{1}}$. So $\left(A^{-1} y\right)(t)$ is a positive $\omega$-periodic solution of (1.3). 
Case 2. If $\underline{f}_{0}>\bar{f}_{\infty}$, in this case, we have

$$
\frac{1-c^{2}}{\bar{f}_{0} l(\alpha-|c|) \int_{0}^{\omega} b(s) \mathrm{d} s}<\lambda<\frac{m-(M+m)|c|}{\underline{f}_{-} L M \int_{0}^{\omega} b(s) \mathrm{ds}} .
$$

It is easy to see that there exists a $0<\varepsilon<f_{0}$ such that

$$
\frac{1-c^{2}}{\left(\bar{f}_{0}-\varepsilon\right) l(\alpha-|c|) \int_{0}^{\omega} b(s) \mathrm{ds}}<\lambda<\frac{m-(M+m)|c|}{\left(\underline{f}_{\infty}+\varepsilon\right) L M \int_{0}^{\omega} b(s) \mathrm{ds}} .
$$

For the above $\varepsilon$, we choose $\bar{r}_{1}>0$ such that $f(u) \geq\left(\bar{f}_{0}-\varepsilon\right) u$ for $0 \leq u \leq \bar{r}_{1}$. Letting $r_{1}=$ $(1-|c|) \bar{r}_{1}$, we have $f\left(\left(A^{-1} y\right)(t-\tau(t))\right) \geq\left(\bar{f}_{0}-\varepsilon\right)\left(A^{-1} y\right)(t-\tau(t))$ for $y \in K_{r_{1}}$. By Lemma 2.2, we have $0 \leq\left(A^{-1} y\right)(t-\tau(t)) \leq\|y\| /(1-|c|) \leq \bar{r}_{1}$ for $y \in \partial K_{r_{1}}$. Thus we have by Lemma 4.3 that for $y \in \partial K_{r_{1}}$

$$
\|Q y\| \geq \lambda l\left(\bar{f}_{0}-\varepsilon\right) \frac{\alpha-|c|}{1-c^{2}} \int_{0}^{\omega} b(s) \mathrm{ds}\|\mathrm{y}\|>\|\mathrm{y}\| .
$$

On the other hand, there exists a constant $\widetilde{H}>0$ such that $f(u) \leq(f+\varepsilon) u$ for $u \geq \widetilde{H}$. Letting $r_{2}=\max \left\{2 r_{1}, \widetilde{H}\left(1-c^{2}\right) /(\alpha-|c|)\right\}$, we have $f\left(\left(A^{-1} y\right)(t-\tau(t))\right) \leq\left(\underline{f}_{-\infty}+\varepsilon\right)\left(A^{-1} y\right)(t-\tau(t))$ for $y \in K_{r_{2}}$. By Lemma 2.2 we have $\left(A^{-1} y\right)(t-\tau(t)) \geq\left((\alpha-|c|) /\left(1-c^{2}\right)\right)\|y\| \geq \widetilde{H}$ for $y \in \partial K_{r_{2}}$. Thus by Lemma 4.4 , for $y \in \partial K_{r_{2}}$,

$$
\|Q y\| \leq \lambda\left(\underline{f}_{\infty}+\varepsilon\right) \frac{L M \int_{0}^{\omega} b(s) \mathrm{ds}}{m-(M+m)|c|}\|y\|
$$

It follows from Lemma 4.7 that

$$
i\left(Q, K_{r_{1}}, K\right)=0, \quad i\left(Q, K_{r_{2}}, K\right)=1
$$

Thus $i\left(Q, K_{r_{2}} \backslash \bar{K}_{r_{1}}, K\right)=-1$ and $Q$ has a fixed point $y$ in $K_{r_{2}} \backslash \bar{K}_{r_{1}}$, proving that $\left(A^{-1} y\right)(t)$ is a positive $\omega$-periodic solution of (1.3).

Case $3\left(\underline{f}_{0} \leq \underline{f}_{\infty} \leq \bar{f}_{0} \leq \bar{f}_{\infty}\right)$. The proof is the same as in Case 1 .

Case $4\left(\underline{f}_{\infty} \leq \underline{f}_{0} \leq \bar{f}_{\infty} \leq \bar{f}_{0}\right)$. The proof is the same as in Case 2 .

4.2. Case II: $c>0$ and $c<\min \{m /(M+m),(L M-l m) /((L-l) M-l m)\}$

Define

$$
f_{2}(r)=\min \left\{f(t): \frac{\alpha}{1-c} r \leq t \leq \frac{r}{1-c}\right\}
$$


Similarly as in Section 4.1, we get the following results.

Theorem 4.11. (a) If $\bar{i}_{0}=1$ or 2 , then (1.3) has $i_{0}$ positive w-periodic solutions for $\lambda>$ $1 / f_{2}(1) l \int_{0}^{\omega} b(s) \mathrm{ds}>0$.

(b) If $\underline{i}_{-\infty}=1$ or 2 , then (1.3) has $i_{\infty}$ positive w-periodic solutions for $0<\lambda<(m-(M+$ $m) c) / L M(1-c) F(1) \int_{0}^{\omega} b(s) \mathrm{ds}$.

(c) If $\bar{i}_{\infty}=0$ or $\underline{i}_{0}=0$, then (1.3) has no positive w-periodic solution for sufficiently small or large $\lambda>0$, respectively.

Theorem 4.12. (a) If there exists a constant $b_{1}>0$ such that $f(u) \geq b_{1} u$ for $u \in[0,+\infty)$, then (1.3) has no positive $\omega$-periodic solution for $\lambda>(1-c) / l \alpha b_{1} \int_{0}^{\omega} b(s) \mathrm{ds}$.

(b) If there exists a constant $b_{2}>0$ such that $f(u) \leq b_{2} u$ for $u \in[0,+\infty)$, then (1.3) has no positive $\omega$-periodic solution for $0<\lambda<(m-(M+m) c) / b_{2} L M \int_{0}^{\omega} b(s) \mathrm{ds}$.

Theorem 4.13. Assume that $\underline{i}_{0}=\bar{i}_{0}=\underline{i}_{-\infty}=\bar{i}_{\infty}=0$ hold, and that one of the following conditions holds:
(1) $\bar{f}_{0} \leq \underline{f}_{\infty} ;$
(2) $\underline{f}_{0}>\bar{f}_{\infty}$;
(3) $\underline{f}_{0} \leq \underline{f}_{\infty} \leq \bar{f}_{0} \leq \bar{f}_{\infty}$;
(4) $\underline{f}_{-\infty} \leq \underline{f}_{0} \leq \bar{f}_{\infty} \leq \bar{f}_{0}$.

If

$$
\frac{1-c}{l \alpha \int_{0}^{\omega} b(s) \mathrm{ds} \max \left\{\underline{\mathrm{f}}_{0}, \overline{\mathrm{f}}_{0}, \underline{\mathrm{f}}_{-\infty}, \overline{\mathrm{f}}_{\infty}\right\}}<\lambda<\frac{m-(M+m) c}{\operatorname{LM} \int_{0}^{\omega} b(s) \mathrm{ds} \min \left\{\underline{\mathrm{f}}_{0}, \overline{\mathrm{f}}_{0}, \underline{\mathrm{f}}_{-\infty}, \overline{\mathrm{f}}_{\infty}\right\}}
$$

then (1.3) has one positive w-periodic solution.

Remark 4.14. In a similar way, one can consider the third-order neutral functional differential equation $(x(t)-c x(t-\delta(t)))^{\prime \prime \prime}-a(t) x(t)=-\lambda b(t) f(x(t-\tau(t)))$.

We illustrate our results with an example.

Example 4.15. Consider the following third-order neutral functional differential equation:

$$
\left(u(t)+\frac{1}{300} u\left(t-\cos ^{2} t\right)\right)^{\prime \prime \prime}+\frac{1}{1000}\left(1-\frac{1}{2} \sin ^{2} t\right) u(t)=\lambda\left(1-\cos ^{2} t\right) u^{2}(t-\tau(t)) a^{u(t-\tau(t))},
$$

where $\lambda$ and $0<a<1$ are two positive parameters, and $\tau(t+\pi)=\tau(t)$.

Comparing (4.60) to (1.3), we see that $\delta(t)=\cos ^{2} t, c=-1 / 300, a(t)=(1 / 1000)(1-$ $\left.(1 / 2) \sin ^{2} t\right), b(t)=1-\cos ^{2} t, \omega=\pi, f(u)=u^{2} a^{u}$. Clearly, $M=1 / 1000, m=1 / 2000$, and we get $\rho=1 / 10$, noticing that $\sqrt{3} \pi / 10<4 \pi / 3$ holds. Moreover, we know that $\bar{f}_{0}=0, \bar{f}_{\infty}=0, \bar{i}_{0}=2$. 
By Theorem 4.8, we easily get the following conclusion: (4.60) has two positive $\omega$-periodic solutions for $\lambda>25 / 1132 \pi r_{1}$, where $r_{1}=\min \{f(0.0027), f(300 / 299)\}$.

In fact, by simple computations, we have

$$
\begin{gathered}
l=\frac{1}{3 \rho(\exp (\rho \omega)-1)}=90.3612, \quad L=\frac{3+2 \exp (-\rho \omega / 2)}{3 \rho^{2}(1-\exp (-\rho \omega / 2))^{2}}=7435.98 \\
k=7.5715, \quad k_{1}=0.0060, \quad \alpha=0.0061 \\
|c|=\frac{1}{300}<\min \left\{k_{1}, \frac{m}{M+m}\right\}=0.0060, \quad|c|=\frac{1}{300}<0.0061=\alpha, \\
f_{1}(1)=\min \left\{f(t): 0.0027 \approx \frac{0.0061-1 / 300}{1-(1 / 300)^{2}} \leq t \leq \frac{300}{299}\right\}=\min \left\{f(0.0027), f\left(\frac{300}{299}\right)\right\}=r_{1}, \\
\frac{1}{f_{1}(1) l \int_{0}^{\omega} b(s) \mathrm{d} s}=\frac{25}{1132 \pi r_{1}} .
\end{gathered}
$$

\section{Lyapunov Stability}

When $\delta(t) \equiv \delta$, then (1.3) is transformed into

$$
(x(t)-c x(t-\delta))^{\prime \prime \prime}=-a(t) x(t)+\lambda b(t) f(x(t-\tau(t))) .
$$

Define operator $A_{1}: X \rightarrow X$ by $\left(A_{1} x\right)(t)=(x(t)-c x(t-\delta))$. Obviously, $A_{1}$ satisfies Lemma 2.1. Denote by $\tau_{*}$ and $\tau^{*}$ the essential infimum and supremum of a given function $\tau \in C^{1}[0, \omega]$, if they exist.

Now, we study the Lyapunov stability of the periodic solutions of (5.1).

Theorem 5.1. Assume $\tau(t)>0$ and $\tau^{\prime}(t)<1$ hold. And the following condition hold:

$\left(F_{1}\right)$ there exists a positive constant $\gamma$ such that

$$
\left|f_{u}(u)\right| \leq r, \quad \forall u \in \mathbb{R}, \text { here } f_{u}=\frac{d f}{d u}
$$

Then every w-periodic solution of (5.1) is Lyapunov stable.

Proof. Letting

$$
z_{1}(t)=x(t), \quad z_{2}(t)=x^{\prime}(t), \quad z_{3}(t)=x^{\prime \prime}(t)
$$


then system (5.1) can be transformed into

$$
\begin{gathered}
z_{1}^{\prime}(t)=z_{2}(t) \\
z_{2}^{\prime}(t)=z_{3}(t) \\
z_{3}^{\prime}(t)=A_{1}^{-1}\left[-a(t) z_{1}(t)+\lambda b(t) f\left(z_{1}(t-\tau(t))\right)\right] .
\end{gathered}
$$

Suppose now that $z^{*}(t)=\left(z_{1}^{*}(t), z_{2}^{*}(t), z_{3}^{*}(t)\right)^{\top}$ is a $\omega$-periodic solution of (5.4). Let $z(t)=\left(z_{1}(t), z_{2}(t), z_{3}(t)\right)^{\top}$ be any arbitrary solution of (5.4). For any $k=1,2,3$, write $w_{k}(t)=$ $z_{k}(t)-z_{k}^{*}(t)$. Then it follows from (5.4) that

$$
\begin{gathered}
w_{1}^{\prime}(t)=w_{2}(t), \\
w_{2}^{\prime}(t)=w_{3}(t), \\
w_{3}^{\prime}(t)=A_{1}^{-1}\left(-a(t)\left(z_{1}(t)-z_{1}^{*}(t)\right)+\lambda b(t)\left(f\left(z_{1}(t-\tau(t))\right)-f\left(z_{1}^{*}(t-\tau(t))\right)\right)\right),
\end{gathered}
$$

and so

$$
\begin{gathered}
\left|w_{1}^{\prime}(t)\right|=\left|w_{2}(t)\right| \\
\left|w_{2}^{\prime}(t)\right|=\left|w_{3}(t)\right| \\
\left|w_{3}^{\prime}(t)\right|=\mid A_{1}^{-1}\left(-a(t)\left(z_{1}(t)-z_{1}^{*}(t)\right)+\lambda b(t)\left(f\left(z_{1}(t-\tau(t))\right)-f\left(z_{1}^{*}(t-\tau(t))\right)\right) \mid .\right.
\end{gathered}
$$

Letting $u_{k}^{(l)}(t)=\left|w_{k}^{(l)}(t)\right|, l=0,1, k=1,2,3$, then

$$
\begin{gathered}
u_{1}^{\prime}(t)=u_{2}(t), \\
u_{2}^{\prime}(t)=u_{3}(t), \\
u_{3}^{\prime}(t)=\mid A_{1}^{-1}\left(-a(t)\left(z_{1}(t)-z_{1}^{*}(t)\right)+\lambda b(t)\left(f\left(z_{1}(t-\tau(t))\right)-f\left(z_{1}^{*}(t-\tau(t))\right)\right) \mid .\right.
\end{gathered}
$$

Take $\beta=\max \left\{\left(|a|_{0}+\lambda|b|_{0} \gamma\right) /|1-| c||, \ln \left(1 /\left(1-\tau^{\prime *}\right)\right) / \tau_{*}, 1\right\}+1$, here $|a|_{0}=\max _{t \in[0, \omega]}|a(t)|$, and define a function $V(\cdot)$ by

$$
V\left(t, u_{1}, u_{2}, u_{3}\right):=e^{-\beta t}\left(u_{1}+u_{2}+u_{3}\right)+\frac{\lambda|b|_{0} \gamma}{|1-| c||} \int_{t-\tau(t)}^{t} e^{-\beta s} u_{1}(s) \mathrm{d} s
$$


There exists a sufficiently small positive constant $\varepsilon$ such that $e^{-\beta t} \geq \varepsilon$. Let $U\left(u_{1}, u_{2}, u_{3}\right)=$ $\varepsilon \sum_{k=1}^{3} u_{k}(t)$. It is obvious that $V\left(t, u_{1}, u_{2}, u_{3}\right)>0$ and $V\left(t, u_{1}, u_{2}, u_{3}\right) \geq U\left(u_{1}, u_{2}, u_{3}\right)>0$. From $\left(F_{1}\right)$ and Lemma 2.1, we get

$$
\begin{aligned}
\dot{V}\left(t, u_{1}, u_{2}, u_{3}\right)= & -\beta e^{-\beta t}\left(u_{1}(t)+u_{2}(t)+u_{3}(t)\right)+e^{-\beta t}\left(u_{2}(t)+u_{3}(t)\right) \\
& +e^{-\beta t}\left|A_{1}^{-1}\left(-a(t)\left(z_{1}(t)-z_{1}^{*}(t)\right)+\lambda b(t)\left(f\left(z_{1}(t-\tau(t))\right)-f\left(z_{1}^{*}(t-\tau(t))\right)\right)\right)\right| \\
& +\frac{\lambda|b|_{0} \gamma}{|1-| c||}\left(e^{-\beta t} u_{1}(t)-e^{-\beta(t-\tau(t))}\left(1-\tau^{\prime}(t)\right) u_{1}(t-\tau(t))\right) \\
\leq & -\beta e^{-\beta t}\left(u_{1}(t)+u_{2}(t)+u_{3}(t)\right)+e^{-\beta t}\left(u_{2}(t)+u_{3}(t)\right) \\
& +\frac{e^{-\beta t}}{|1-| c||}\left[|a(t)|\left|z_{1}(t)-z_{1}^{*}(t)\right|+\lambda|b(t)| \gamma\left|\left(z_{1}(t-\tau(t))\right)-z_{1}^{*}(t-\tau(t))\right|\right] \\
& +\frac{\lambda|b|_{0} \gamma}{|1-| c||}\left(e^{-\beta t} u_{1}(t)-e^{-\beta(t-\tau(t))}\left(1-\tau^{\prime}(t)\right) u_{1}(t-\tau(t))\right) \\
\leq & \left(-\beta+\frac{|a|_{0}+\lambda|b|_{0} \gamma}{|1-| c||}\right) e^{-\beta t} u_{1}(t)+e^{-\beta t}(-\beta+1)\left(u_{2}(t)+u_{3}(t)\right) \\
& +\frac{\lambda|b|_{0} \gamma}{|1-| c||}\left(e^{-\beta t}-e^{-\beta(t-\tau(t))}\left(1-\tau^{\prime}(t)\right)\right) u_{1}(t-\tau(t)) \\
= & \left(-\beta+\frac{|a|_{0}+\lambda|b|_{0} \gamma}{|1-| c||}\right) e^{-\beta t} u_{1}(t)+e^{-\beta t}(-\beta+1)\left(u_{2}(t)+u_{3}(t)\right) \\
& +\frac{\lambda|b|_{0} \gamma}{|1-| c||} e^{-\beta t}\left(1-e^{\beta \tau_{*}}\left(1-\tau^{\prime *}\right)\right) u_{1}(t-\tau(t)) \\
& +\frac{\lambda|b|_{0} \gamma}{|1-| c||} e^{-\beta t}\left(1-e^{\beta \tau(t)}\left(1-\tau^{\prime}(t)\right)\right) u_{1}(t-\tau(t)) \\
\leq & \left(-\beta+\frac{|a|_{0}+\lambda|b|_{0} \gamma}{|1-| c||}\right) e^{-\beta t} u_{1}(t)+e^{-\beta t}(-\beta+1)\left(u_{2}(t)+u_{3}(t)\right) \\
& (-\beta)
\end{aligned}
$$

Hence $V$ is a Lyapunov function for nonautonomous (5.1) (see [14, page 50]), and so the $\omega$-periodic solution $z^{*}$ of (5.1) is Lyapunov stable.

Corollary 5.2. Assume that $\tau(t)=\tau>0$ and $\left(F_{1}\right)$ holds. Then every w-periodic solution of (5.1) is Lyapunov stable.

We illustrate our results with an example. 
Example 5.3. Consider the following third-order neutral functional differential equation:

$$
\left(u(t)+\frac{1}{500} u(t-\delta)\right)^{\prime \prime \prime}+\frac{1}{8}\left(1-\frac{1}{2} \sin ^{2} t\right) u(t)=\lambda\left(1-\cos ^{2} t\right)\left(a u(t-\tau)+\cos ^{2} u(t-\tau)\right),
$$

where $\lambda, \delta, \tau$, and $a$ are positive parameters.

Comparing (5.10) to (5.1), we see that $c=-1 / 500, a(t)=(1 / 8)\left(1-(1 / 2) \sin ^{2} t\right), b(t)=$ $1-\cos ^{2} t, \omega=\pi, f(u)=a u+\cos ^{2} u$. Clearly, $M=1 / 8, m=1 / 16$, and we get $\rho=1 / 2$. Noticing that $\sqrt{3} \pi / 2<4 \pi / 3$ holds. Moreover, we know that $f_{0}=\infty, f=a, \underline{i}_{-\infty}=1$. By Theorem 4.8, we easily get the following conclusion: (5.10) has at least one positive $\omega$-periodic solutions for $0<\lambda<1 / 25 \pi r_{2}$, where $r_{2}=\min \{f(0), f(500 / 499)\}$.

In fact, by simple computations, we have

$$
\begin{gathered}
l=\frac{1}{3 \rho(\exp (\rho \omega)-1)}=0.175, \quad L=\frac{3+2 \exp (-\rho \omega / 2)}{3 \rho^{2}(1-\exp (-\rho \omega / 2))^{2}}=17.62, \\
k=2.235, \quad k_{1}=0.0050, \quad \alpha=0.0049, \\
|c|=\frac{1}{500}<\min \left\{k_{1}, \frac{m}{M+m}\right\}=0.0050, \quad|c|=\frac{1}{500}<0.0049=\alpha, \\
F(1)=\min \left\{f(t): 0 \leq t \leq \frac{500}{499}\right\}=\min \left\{f(0), f\left(\frac{500}{499}\right)\right\}=r_{2}, \\
\frac{m-(M-m)|c|}{L M(1-|c|) F(1) \int_{0}^{\omega} b(s) \mathrm{d} s} \approx \frac{1}{25 \pi r_{2}} .
\end{gathered}
$$

Next, we consider that solution of (5.10) is Lyapunov stable. Since $\left|f_{u}(u)\right| \leq a+2$, then $\left(F_{1}\right)$ holds. By Corollary 5.2, we know solution of (5.10) is Lyapunov stable.

\section{Acknowledgment}

This research is supported by the National Natural Science Foundation of China (no. 10971202).

\section{References}

[1] Y. Kuang, Delay Differential Equations with Applications in Population Dynamics, vol. 191 of Mathematics in Science and Engineering, Academic Press, Boston, Mass,USA, 1993.

[2] G. W. Evans and G. Ramey, "A daptive expections, underparameterization and the Lucas critique," Journal of Monetary Economics, vol. 53, pp. 249-264, 2006.

[3] J. Hale, Theory of Functional Differential Equations, Springer, New York, NY, USA, 2nd edition, 1977.

[4] Z. X. Zheng, Theory of Functional Differential Equations, Anhui Educational Publishing House, Hefei, China, 1994.

[5] J. Wu and Z. Wang, "Two periodic solutions of second-order neutral functional differential equations," Journal of Mathematical Analysis and Applications, vol. 329, no. 1, pp. 677-689, 2007.

[6] W.-S. Cheung, J. Ren, and W. Han, "Positive periodic solution of second-order neutral functional differential equations," Nonlinear Analysis, vol. 71, no. 9, pp. 3948-3955, 2009. 
[7] S. Lu and W. Ge, "Periodic solutions of neutral differential equation with multiple deviating arguments," Applied Mathematics and Computation, vol. 156, no. 3, pp. 705-717, 2004.

[8] S. $\mathrm{Lu}$ and W. Ge, "Existence of periodic solutions for a kind of second-order neutral functional differential equation," Applied Mathematics and Computation, vol. 157, no. 2, pp. 433-448, 2004.

[9] Q. Wang and B. Dai, "Three periodic solutions of nonlinear neutral functional differential equations," Nonlinear Analysis, vol. 9, no. 3, pp. 977-984, 2008.

[10] Y. Luo and Z. Luo, "Existence of positive periodic solutions for neutral multi-delay logarithmic population model," Applied Mathematics and Computation, vol. 216, no. 4, pp. 1310-1315, 2010.

[11] F. Zhang and C. Zheng, "Positive periodic solutions for the neutral ratio-dependent predatorprey model," Computers and Mathematics with Applications, vol. 61, no. 8, pp. 2221-2226, 2011.

[12] M. R. Zhang, "Periodic solutions of linear and quasilinear neutral functional-differential equations," Journal of Mathematical Analysis and Applications, vol. 189, no. 2, pp. 378-392, 1995.

[13] M. A. Krasnoselskii, Positive Solutions of Operator Equations, Translated from the Russian by Richard E. Flaherty; edited by Leo F. Boron, P. Noordhoff, Groningen, The Netherlands, 1964.

[14] P. Glendinning, Stability, Instability and Chaos: An Introduction to the Theory of Nonlinear Differential Equations, Cambridge Texts in Applied Mathematics, Cambridge University Press, Cambridge, UK, 1994. 


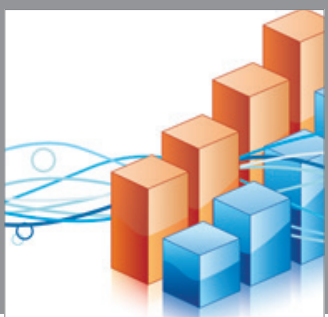

Advances in

Operations Research

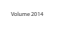

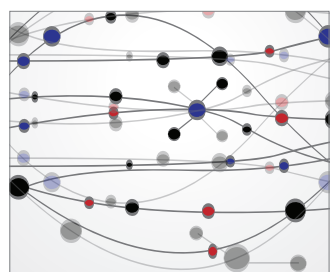

\section{The Scientific} World Journal
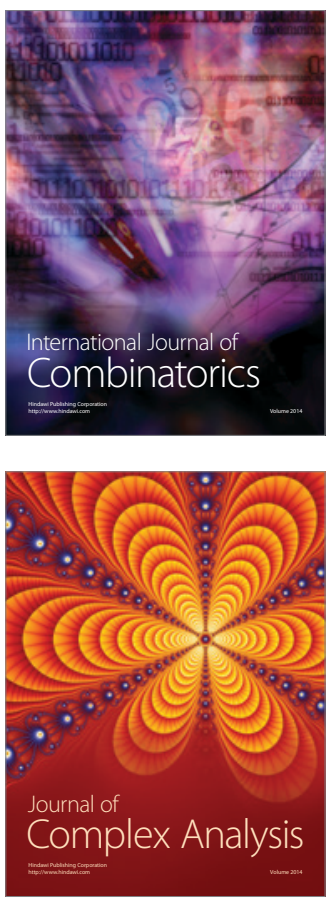

International Journal of

Mathematics and

Mathematical

Sciences
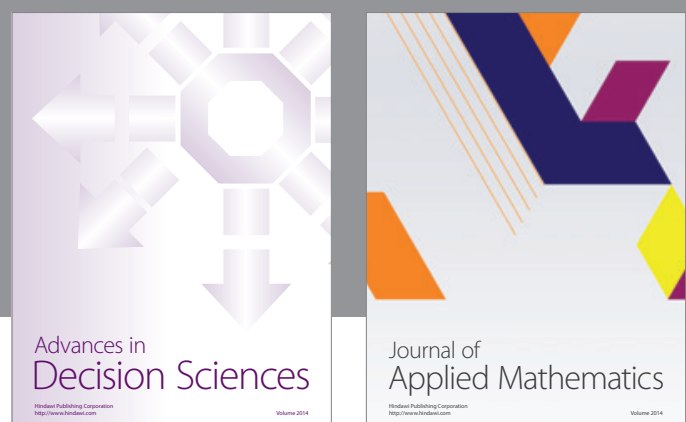

Journal of

Applied Mathematics
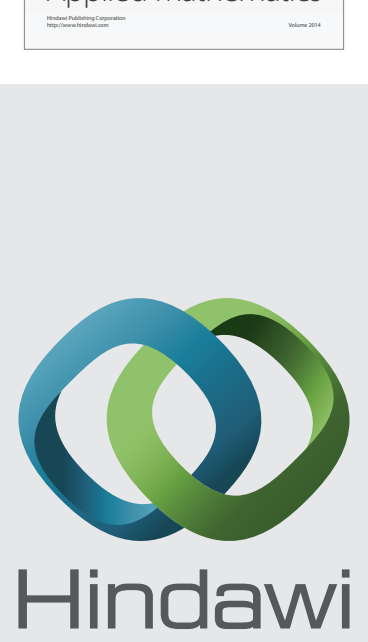

Submit your manuscripts at http://www.hindawi.com
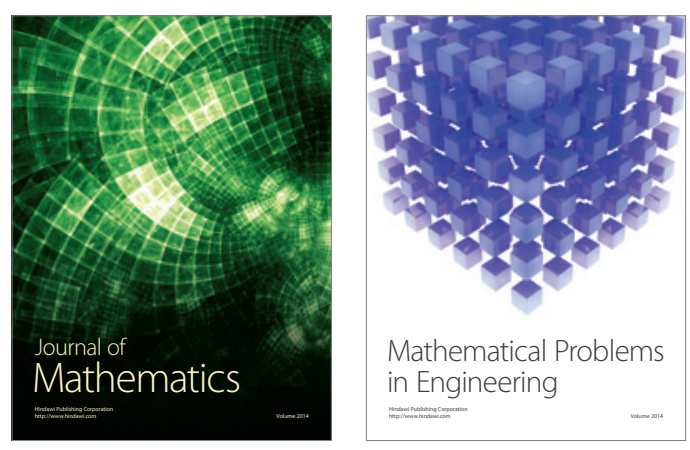

Mathematical Problems in Engineering
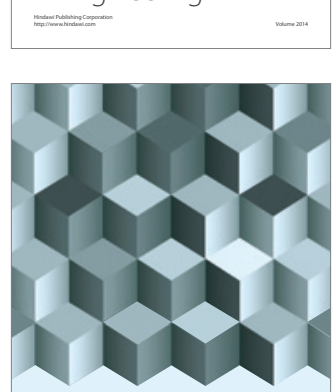

Journal of

Function Spaces
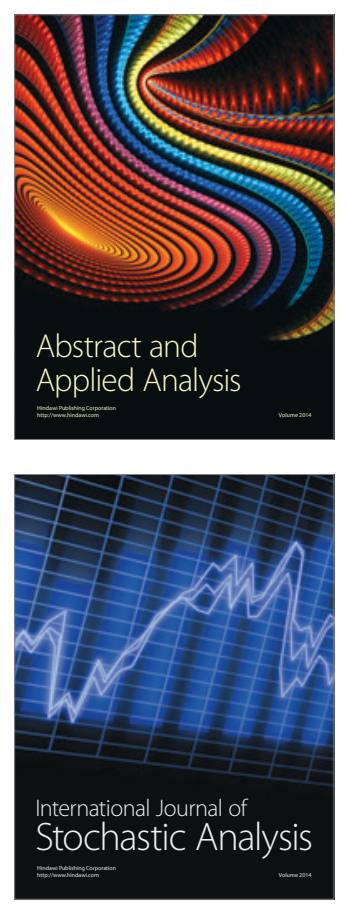

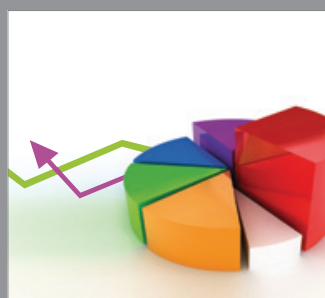

ournal of

Probability and Statistics

Promensencen
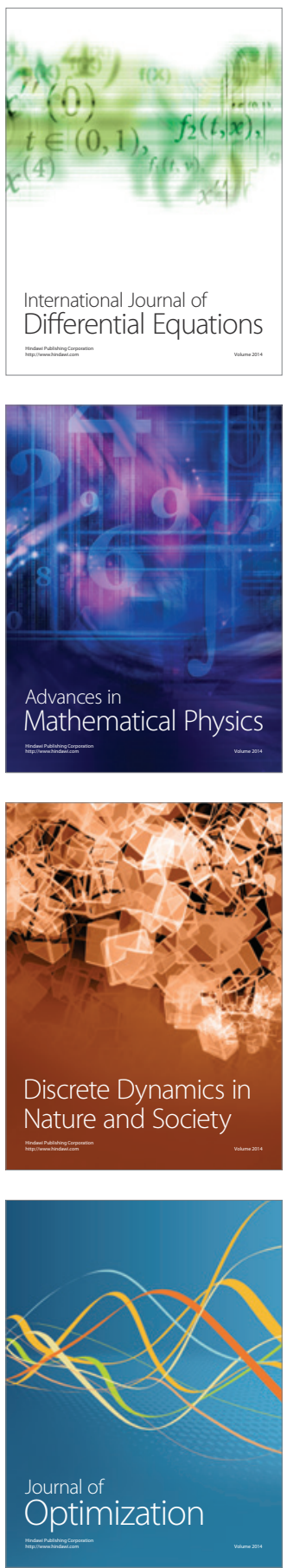